\title{
A recyclable nanocarbon white emitter via the synergy between carbon dots and organic sheet
}

Yamei Ding, ${ }^{\dagger} \S$ Shuai Cheng, ${ }^{\dagger} \S$ Qing Chang, ${ }^{\dagger}$ Shang Ju, ${ }^{\dagger}$ Chaoyi Ban,${ }^{\dagger}$ Zhe Zhou, ${ }^{\dagger}$ Huiwu Mao, ${ }^{\dagger}$ Juqing Liu, ${ }^{*}, \dagger$ Fei Xiu, ${ }^{*}, \dagger$ Wei Huang ${ }^{*}, \dagger, \star, \perp$

†Key Laboratory of Flexible Electronics (KLOFE) \& Institute of Advanced Materials (IAM), Nanjing Tech University (NanjingTech), 30 South Puzhu Road, Nanjing 211816, China.

$¥$ Key Laboratory for Organic Electronics and Information Displays \& Institute of Advanced Materials (IAM), SICAM, Nanjing University of Posts \& Telecommunications, 9 Wenyuan Road, Nanjing 210023, China.

$\perp$ Shaanxi Institute of Flexible Electronics (SIFE), Northwestern Polytechnical University (NPU), 127 West Youyi Road, Xi'an 710072, China.

Number of Pages: 17

Number of Figures: 16 

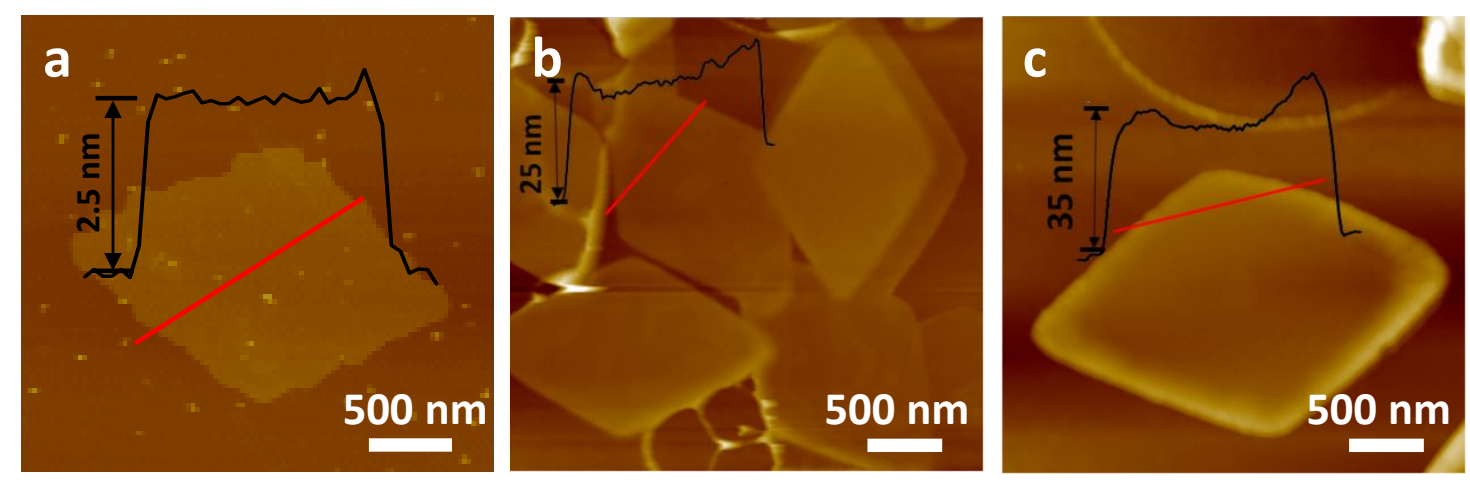

Figure S1. AFM images of nanosheets with an average thickness of $2.5 \mathrm{~nm}$ (a), $25 \mathrm{~nm}$ (b), and $35 \mathrm{~nm}$ (c), promoted by $\mathrm{H}-\mathrm{CDs}$, D-CDs, and P-CDs, respectively. 


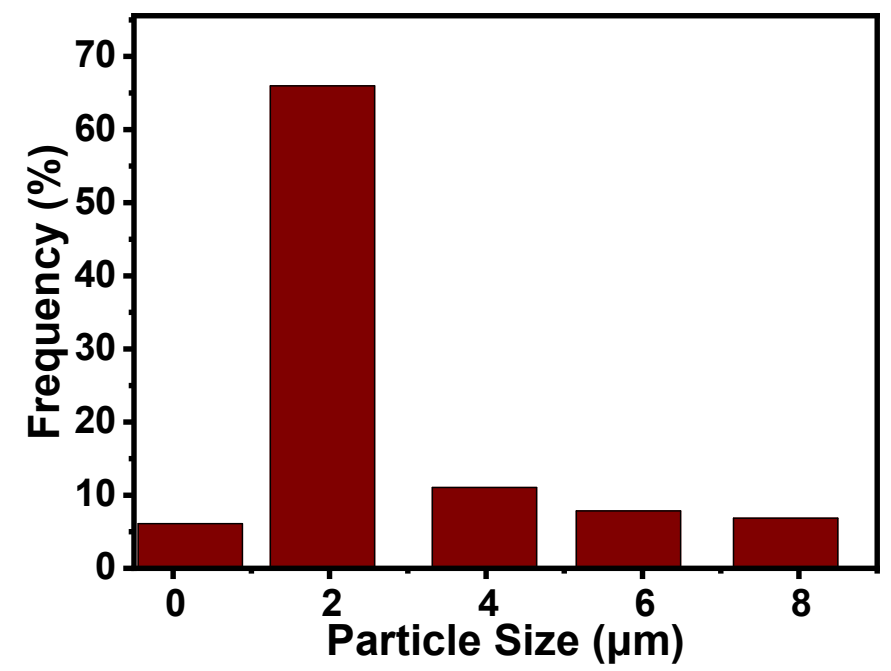

Figure S2. Particle size distribution of organic nanosheets measured by dynamic light scattering (DLS). The majority particle size of nanosheets ranges from 1 to $3 \mu \mathrm{m}$, which is consistent with the SEM results, indicating that carbon nanosheets are formed in chloroform solution. 

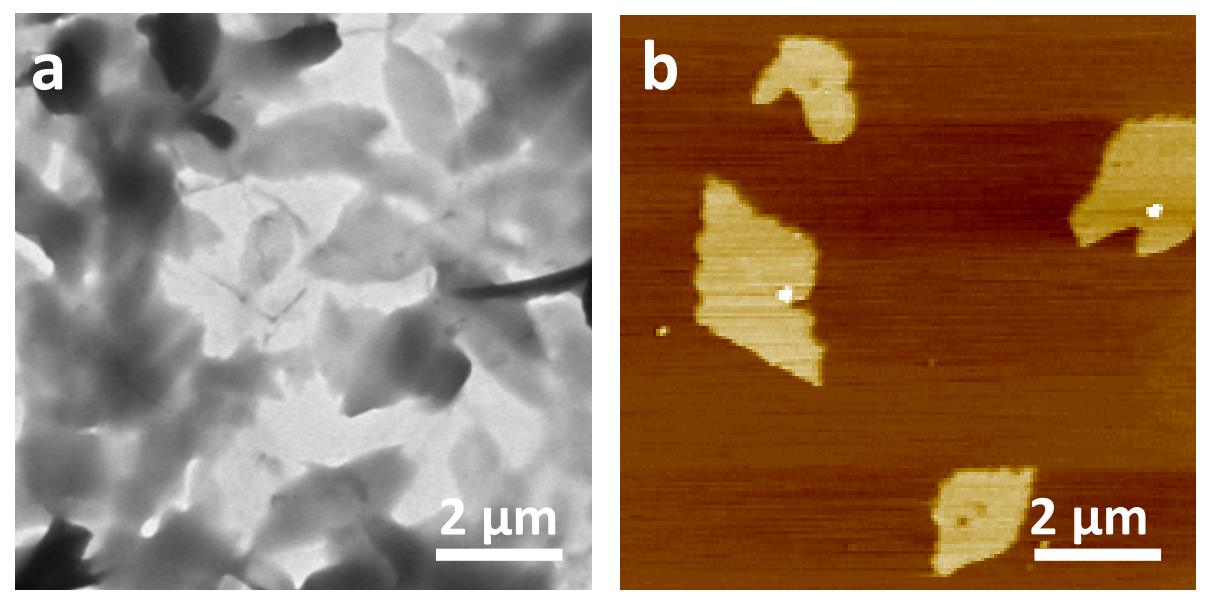

Figure S3. (a) TEM image of products from HDA under the same pyrolytic treatment conditions without the participation of CA. (b) AFM image of organic nanosheets with obvious structural rupture after removing CDs via dialysis for several days.

Atactic organic nanosheets are obtained from the products in which carbon dots have been separated from the prepared nanocarbon emitter solution by dialyzing against chloroform solvent through the dialysis membrane $(\mathrm{Da}=200)$ for several days. This process is finished when the chloroform solution outside the dialysis membrane exhibits no fluorescence. 

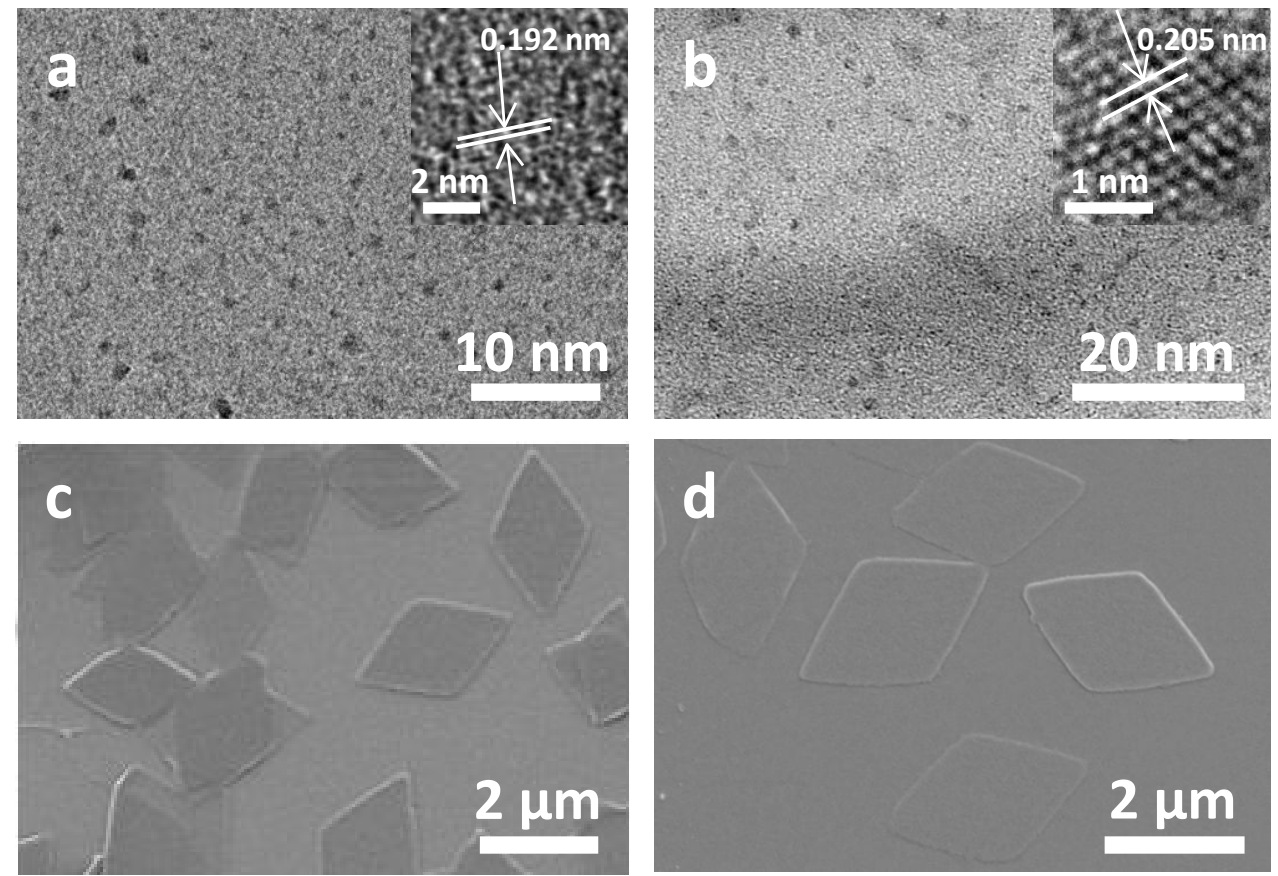

Figure S4. (a, b) TEM and HRTEM (inset) images of D-CDs, and P-CDs. (c, d) SEM images of regular organic nanosheets supported by D-CDs and P-CDs, renewedly. 


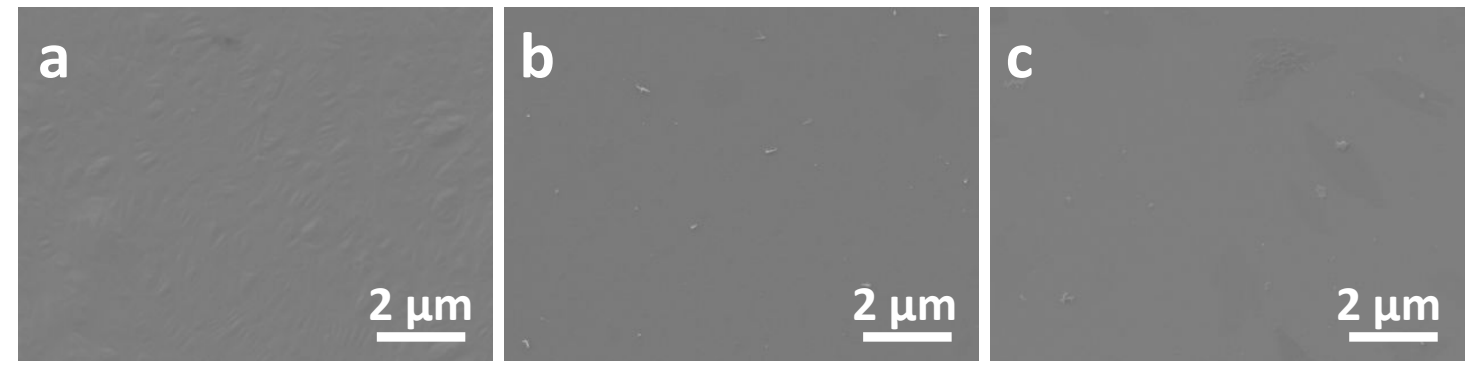

Figure S5. (a-c) SEM images of H-CDs, D-CDs, and P-CDs, respectively. Three kinds of as-prepared carbon dots have no ability to self-assemble in the same condition. It has ruled out the possibility of self-assembly of carbon dots and their carbon precursors. 
a

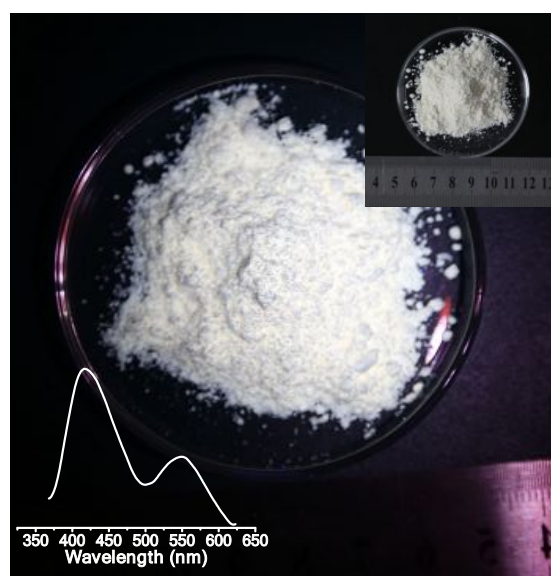

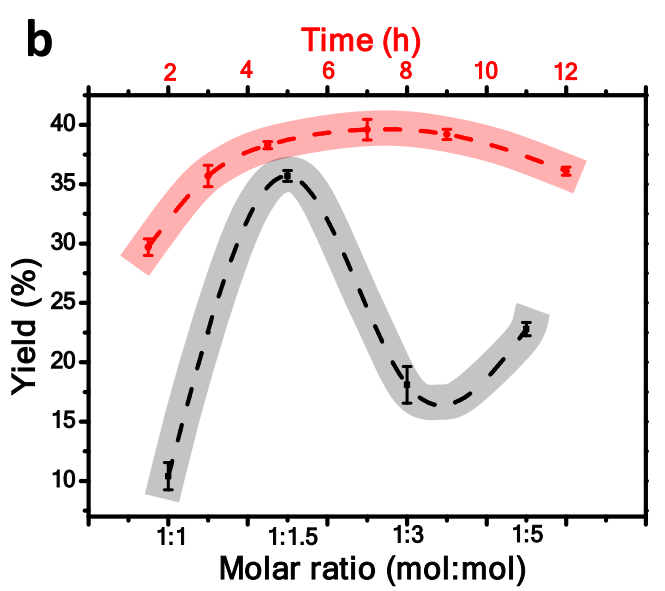

Figure S6. (a) Images of emitter powders under the UV irradiation (365 nm). Top-right inset shows the image of emitter powders in daylight, and the left-bottom inset is PL spectrum of hybrid carbon emitters in the solid state when excited at $365 \mathrm{~nm}$. (b) The yield figure of carbon emitter when the molar ratio of precursor changes (black line) and reaction time varies (red line).

To explore a satisfactory production yield of high quality carbon emitters, reaction conditions including the reaction time and ratio of reactants are optimized (Figure S4b). The reaction time shows a parabolic trend with the optimal reaction time of $7 \mathrm{~h}$. The optimum ratio of CA to HDA is found to be 1:1.5 when the molar ratio of CA and HDA is changed from 1:1 to 1:5. Based on the optimum precursor ratio of 1:1.5 and optimal reaction time of $7 \mathrm{~h}$, a high yield preparation of carbon emitters (40\%) has been realized. 


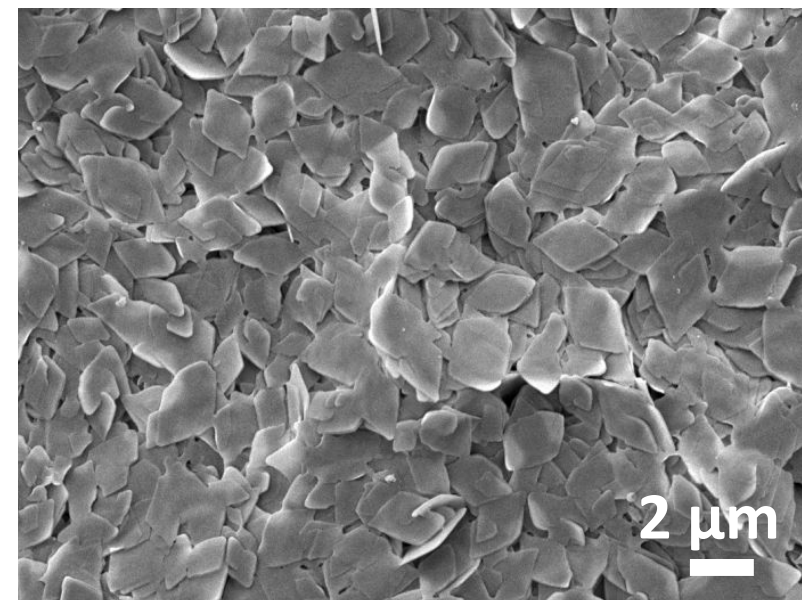

Figure S7. SEM image of low-dimensional dot/sheet nanocarbon emitters with great film-forming ability. 

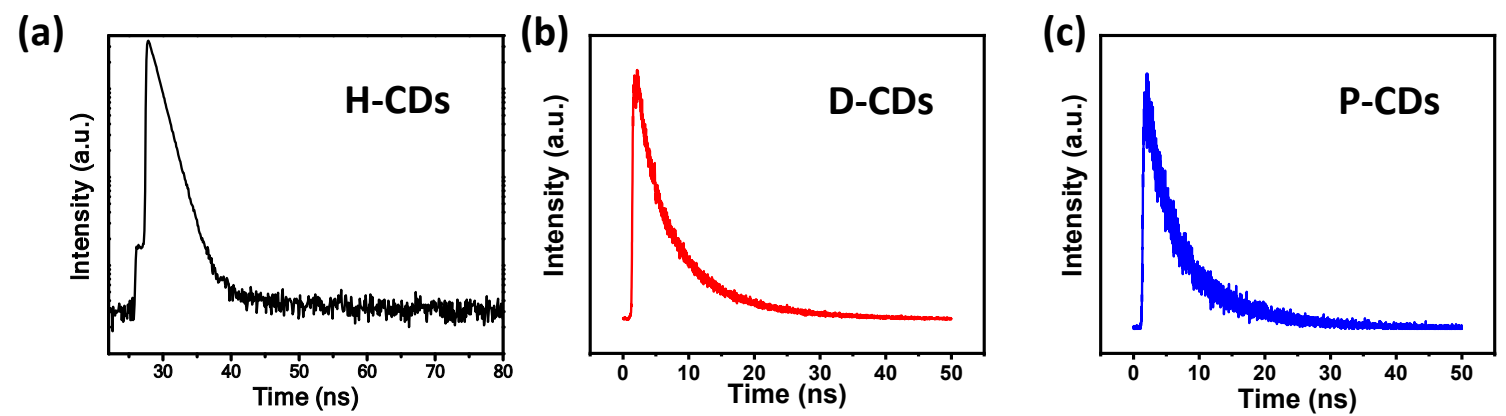

Figure S8. PL decay curve of H-CDs, D-CDs and P-CDs, respectively.

The H-CDs exhibit the bright blue emission with single exponential lifetime decay $(\tau=1.53$ ns). Interestingly, the QY of blue C-dots can reach up to an impressively high value (82\%) at exceptionally high concentrations in solution because of its excellent dispersibility, which is different from common CDs. PL decay curve of D-CDs and P-CDs reveal the resultant average lifetime of $8.5 \mathrm{~ns}$ and $8.8 \mathrm{~ns}$, respectively. The PL QYs are determined to be $55 \%$ and 23\% for D-CDs and P-CDs, which are similar with that of the cited literatures (Angew. Chem. Int. Ed. 2015, 54, 1-5; Adv. Mater. 2017, 29, 16604436). 

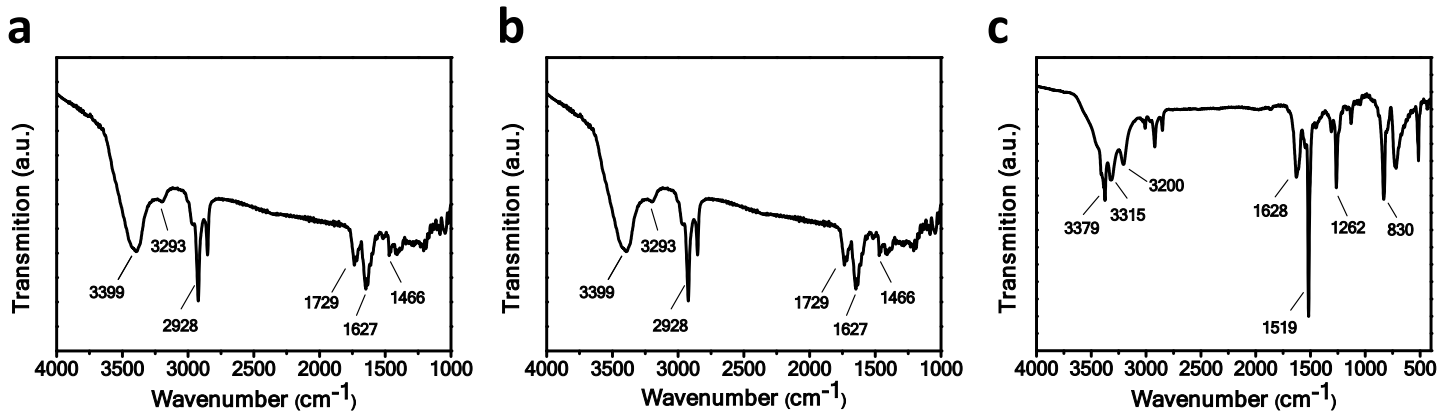

Figure S9. (a-c) FT-IR spectra of H-CDs, D-CDs, and P-CDs, respectively. Surface groups are confirmed from FT-IR spectra of these three CDs. 
a

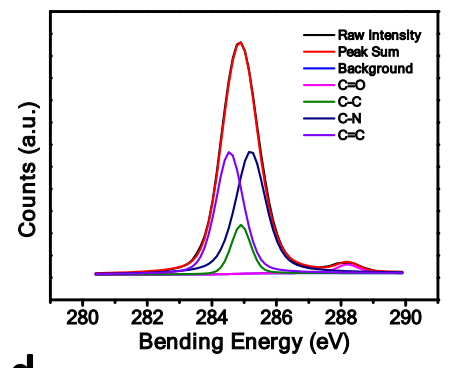

d

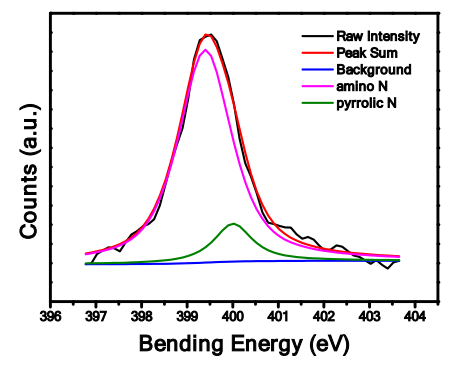

b

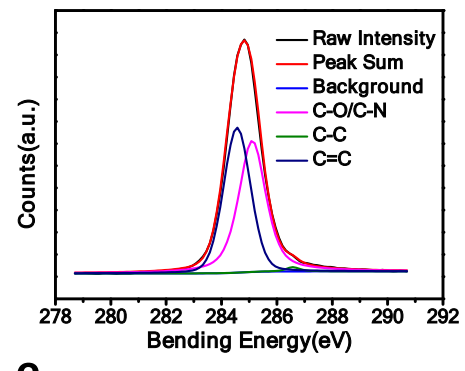

e

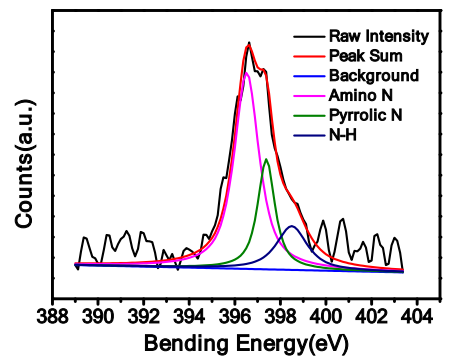

C

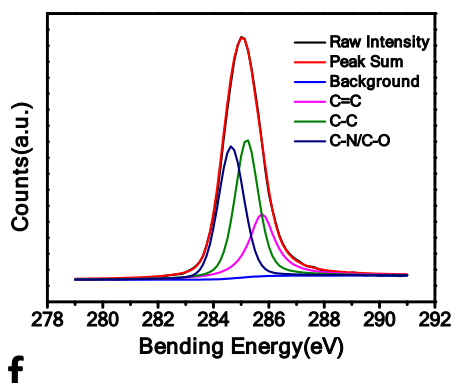

f

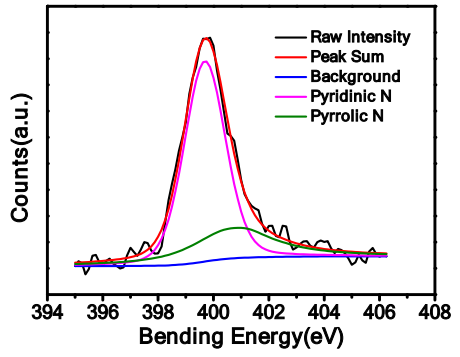

Figure S10. (a-c) Detailed C1s XPS spectra of H-CDs, D-CDs, and P-CDs, respectively. (d-f)

Detailed N1s XPS spectra of H-CDs, D-CDs, and P-CDs, respectively.

For H-CDs, Fig. 2d shows three peaks at 284.2, 398.1, and $528.3 \mathrm{eV}$, corresponding to the $\mathrm{C} 1 \mathrm{~s}, \mathrm{~N} 1 \mathrm{~s}$, and $\mathrm{O} 1 \mathrm{~s}$ peak of $\mathrm{H}-\mathrm{CDs}$, respectively. The elemental analysis reveals the composition of $\mathrm{CDs}$ as follows: $\mathrm{C}, 87.62 \%$;, $3.86 \%$; $\mathrm{O}, 8.70 \%$. In detail, the $\mathrm{C} 1 \mathrm{~s}$ peak is divided into four peaks at $284.5,284.9,285.2$, and $288.2 \mathrm{eV}$, which correspond to $\mathrm{C}=\mathrm{C}, \mathrm{C}-\mathrm{C}$, $\mathrm{C}-\mathrm{N}$, and $\mathrm{C}=\mathrm{O}$, respectively. Moreover, $\mathrm{C} 1$ s peak of $\mathrm{D}-\mathrm{CDs}$ can also be fitted into three peaks at 245.6, 285.1, and $286.6 \mathrm{eV}$ referring to $\mathrm{C}=\mathrm{C}, \mathrm{C}-\mathrm{O} / \mathrm{C}-\mathrm{N}$, and $\mathrm{C}-\mathrm{C}$, respectively. C1s XPS of H-CDs exhibits three peaks at 248.6, 285.2, and $285.8 \mathrm{eV}$, corresponding to C-N/C-O, C-C, and $\mathrm{C}=\mathrm{C}$, respectively.

For $\mathrm{N}$ elements, two peaks at 399.4 and $400.1 \mathrm{eV}$ can be observed due to the existence of amino $\mathrm{N}$ and pyrrolic $\mathrm{N}$ in H-CDs. D-CDs reveals three peaks at 396.5, 397.4, 
and $398.5 \mathrm{eV}$, referring to amine N, pyrrolic N, and N-H. Moreover, N1s of P-CDs can be fitted into 399.7 and $400.8 \mathrm{eV}$, corresponding to pyridinic $\mathrm{N}$ and pyrrolic $\mathrm{N}$, respectively.
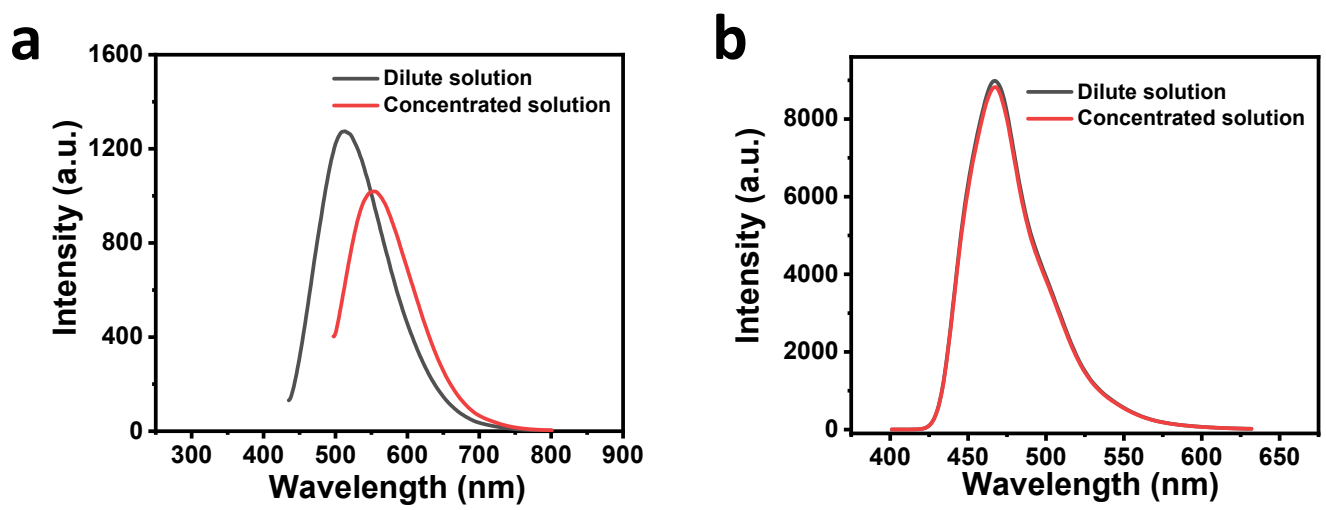

Figure S11. PL spectra of C-dots with different concentrations obtained from CA (a) and the passivated CDs from CA and HDA (b). 
a

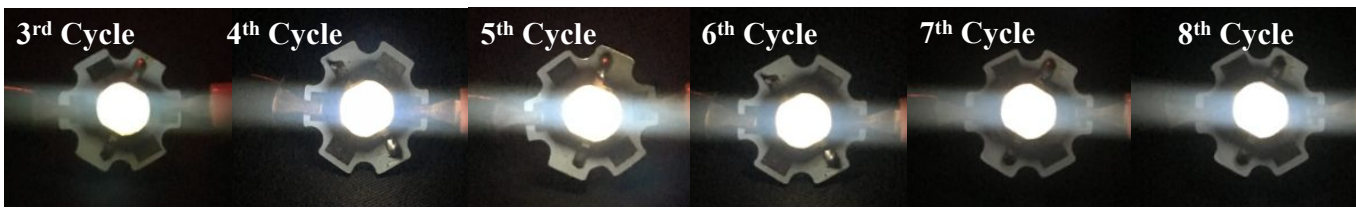

b

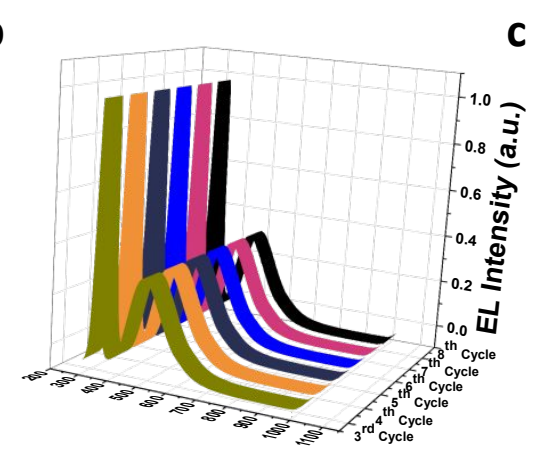

C

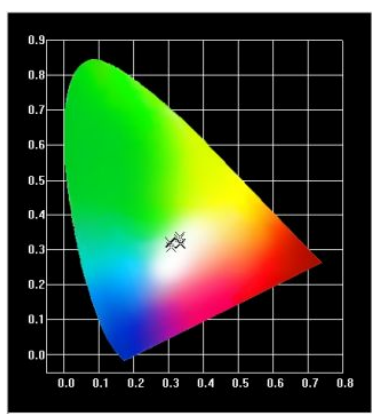

d

\begin{tabular}{c|c} 
Cycle-index & CIE coordinates \\
\hline 3 & $(0.332,0.332)$ \\
\hline 4 & $(0.332,0.317)$ \\
\hline 5 & $(0.329,0.332)$ \\
\hline 6 & $(0.334,0.316)$ \\
\hline 7 & $(0.308,0.320)$ \\
\hline 8 & $(0.325,0.321)$ \\
\hline 9 & $(0.315,0.321)$
\end{tabular}

Figure S12. (a) Digital images of WLEDs coated with as-prepared nanocarbon phosphors for eight times recycling use. (b) EL images of phosphor-coated WLEDs for eight times recycling use. (c,d) The corresponding CIE chromaticity diagrams of eight phosphor-coated WLEDs for recyclable use. 

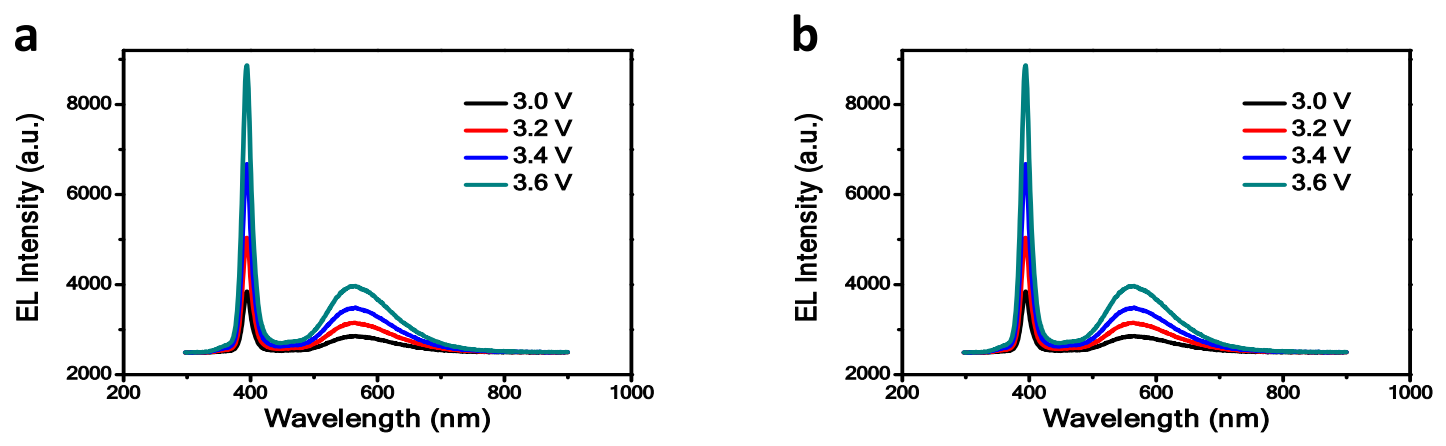

Figure S13. EL spectra of the light emitting device when coated conventional transparent phosphors (a) and as-prepared dot/sheet nanocarbon emitters (b) under different voltages. 

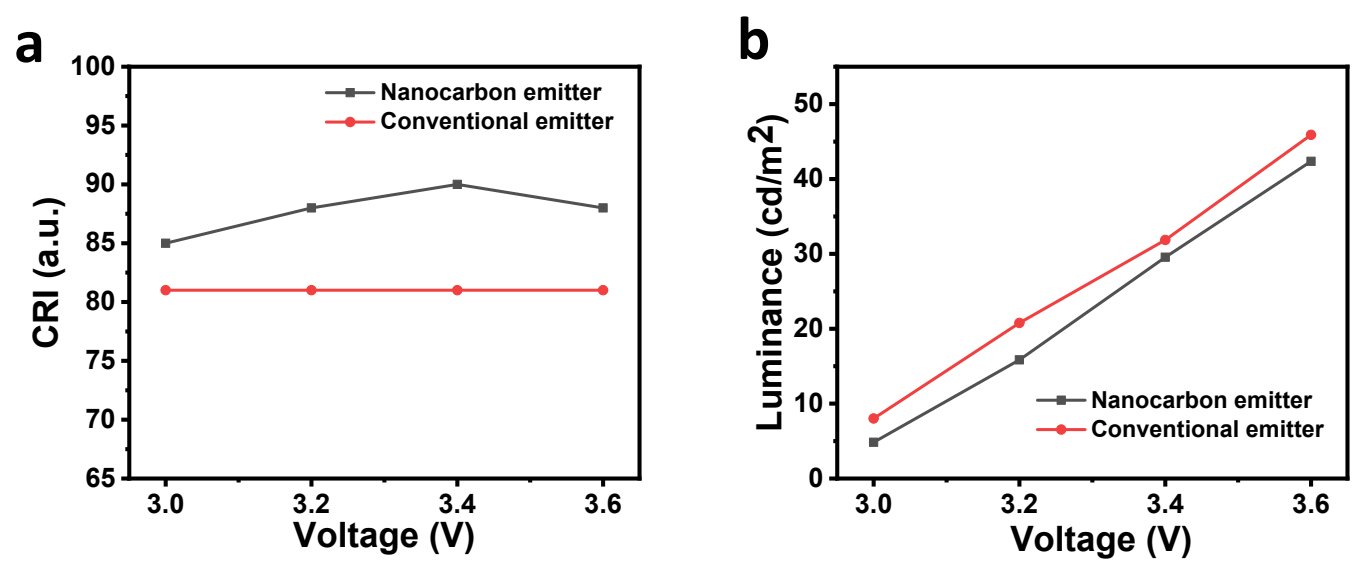

Figure S14. CRI (a) and Luminance changes (b) of LEDs when coating as-prepared emitters and transparent phosphors with increasing voltages.

For more performance of LEDs when coating as-prepared emitters and transparent phosphors, the color rendering index (CRI) of our nanocarbon emitter is higher than conventional transparent phosphors, revealing the great color rendering effect. The luminance of LEDs coated with as-prepared emitters is lower than that of LEDs coated with transparent phosphors at lower voltage but narrows the gap of lumincance with the increasing voltages. 

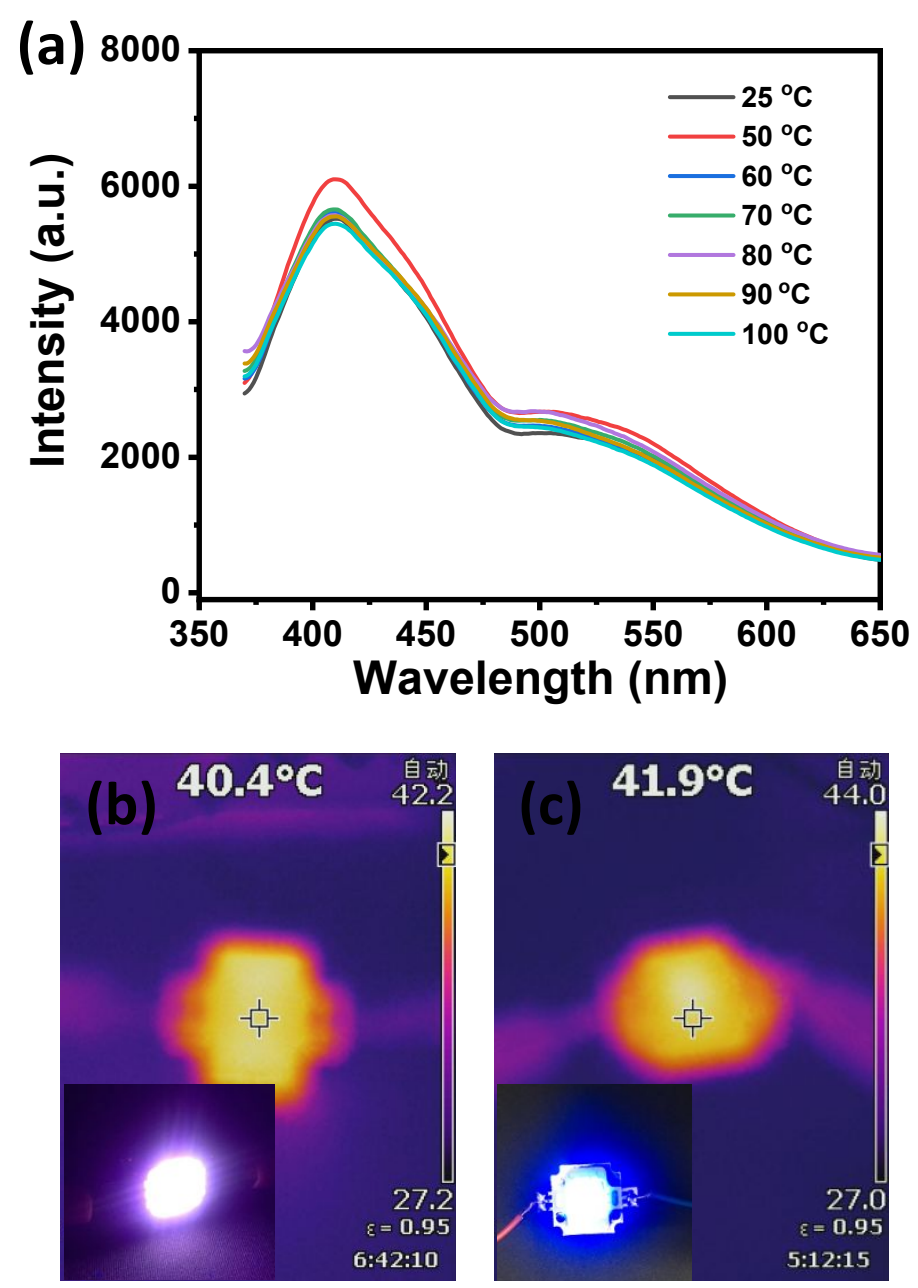

Figure S15. (a) PL spectra of carbon emitters in the solid state after heat treatment at different temperatures. Thermal images of LED chips of $365 \mathrm{~nm}$ (b) and $420 \mathrm{~nm}$ (c) after working ten hours. 


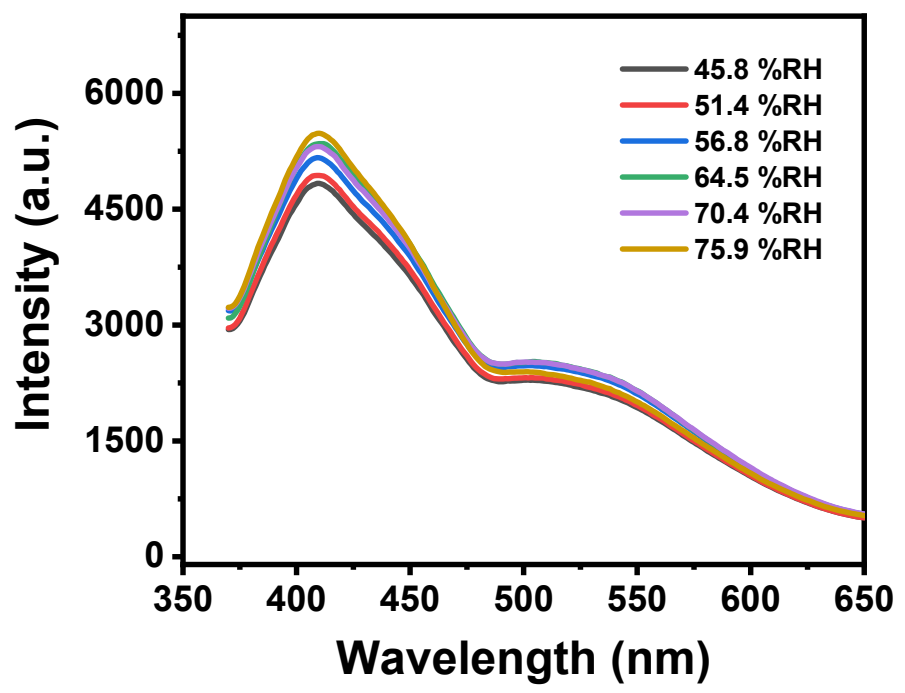

Figure S16. PL spectra of phosphors under different humidity. 